\title{
METHODS OF FUNCTION APPROXIMATION AND MODERN COMPUTER-BASED TECHNOLOGIES (REVIEW)
}

\author{
O. N. Litvin ${ }^{\mathrm{a}}$ and I. V. Sergienko ${ }^{\mathrm{b}}$
}

UDC 519.6

\begin{abstract}
The paper reviews publications over the last five years devoted to efficient solutions of some important practical problems using interlineation and interflation (blending function interpolation) and blending function approximation.
\end{abstract}

Keywords: interlineation, interflation, mapping, tomography, optimization, modern computer-based technology.

\section{INTRODUCTION}

An analysis of the modern trends in the field of computational methods allows us to conclude that more and more researchers use computational methods with functions of two and more variables. Considerable attention is focused on optimizing the methods of approximation of multivariable functions, defined explicitly or being solutions to differential or integral equations. The information used to compose the operators of approximation includes the following: the values of the function being approximated and its partial derivatives up to a fixed order in a given system of points; traces of the function being approximated and traces of the set of differential operators of the functions being approximated (for example, its partial derivatives or its normal derivatives, etc.) up to a fixed order on a given system of lines (for functions of two and more variables) or surfaces (for functions of three and more variables); Radon data (projections) that arrive from a computer tomograph (for example, line integrals along a given system of lines or surfaces with respect to volume density of the body under study); tomograms of sections of the body; satellite photos of some parts of planets or other space objects, etc.

Thus, we may state that digital processing of multidimensional signals that may arrive at the input of a processor and should be processed rather quickly (often in real time) has come to the fore. To evaluate the complexity of the above-mentioned problems, which can efficiently be solved due mainly to modern PCs, we will dwell on the above-mentioned examples.

\section{EXAMPLES OF ENGINEERING PROBLEMS WHOSE OPTIMAL SOLUTION REQUIRES RECOVERY OF FUNCTIONS OF MANY VARIABLES AND MODERN COMPUTER TECHNIQUES}

Example 1. Sonar Seafloor Mapping. Seafloor mapping is based on sonar data (see Fig. 1) obtained along lines, which are the courses of a ship with a sonar.

Assume that $z=f(x, y)$ is the equation describing the seafloor surface. The task is to recover virtually (possibly, approximately) this surface, i.e., the function $f(x, y)$ using the minimum amount of experimental data $f\left(x_{k}, y\right), k=\overline{1, M_{1}} ; f\left(x, y_{l}\right), l=\overline{1, M_{2}}$, or (in the general case) $\left.\varphi_{k}(x, y)\right|_{\Gamma_{k}}=\left.f(x, y)\right|_{\Gamma_{k}}, k=\overline{1, M}$, where $\left\{\Gamma_{k}\right\}$ is a system of lines (ship courses); $M_{1}, M_{2}$, and $M$ are given numbers.

${ }^{\mathrm{a}}$ Ukrainian Engineering and Pedagogical Academy, Kharkov, Ukraine, academ@kharkov.ua. ${ }^{\mathrm{b}} \mathrm{V} . \mathrm{M}$. Glushkov Institute of Cybernetics, National Academy of Sciences of Ukraine, Kyiv, Ukraine, aik@public.icyb.kiev.ua. Translated from Kibernetika i Sistemnyi Analiz, No. 1, pp. 64-81, January-February 2007. Original article submitted September $23,2005$. 


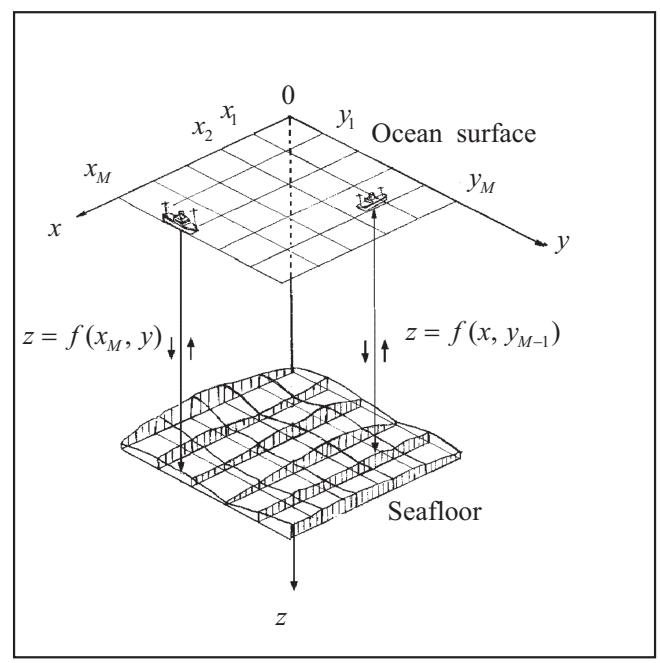

Fig. 1. Sonar data obtained on the lines $x=x_{k} \quad(k=\overline{1, M})$ and $y=y_{i} \quad(i=\overline{1, N})$ (ship courses) on the ocean surface.

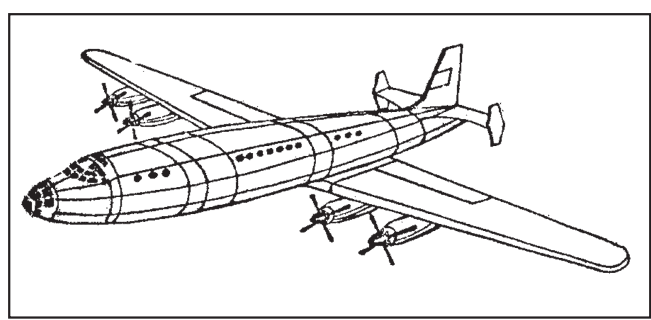

Fig. 2. Airframe consisting of a set of lines to which pieces of surface with required properties are attached.

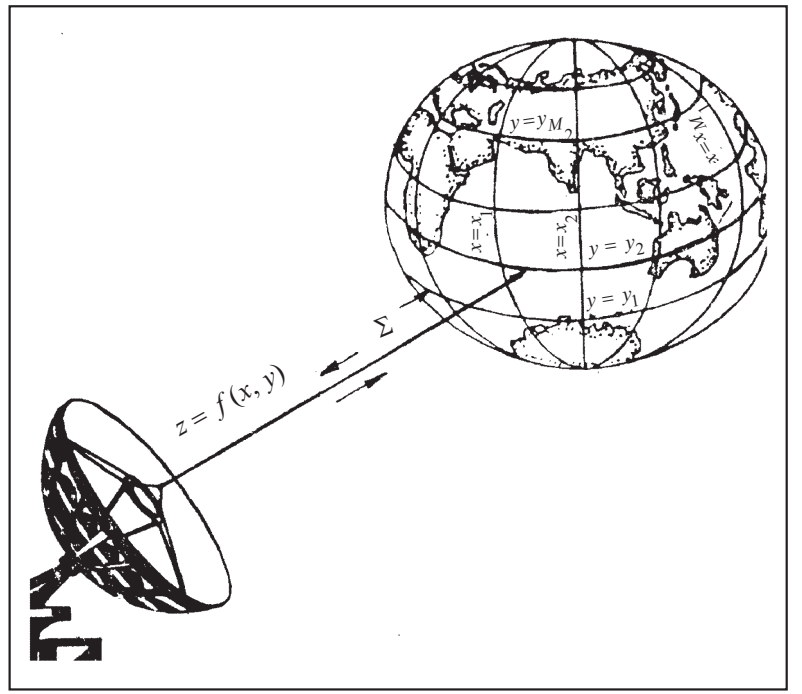

Fig. 3. Radar data are collected along lines on the surface of a space body.

Example 2. Designing Aircraft, Rockets, Ships, and Automobiles. The aircraft surface is formed of skin panels (Fig. 2) attached to the frame. The equations $z=f_{i}(u, v)$ of the $i$ th piece of the surface (in the corresponding parametric representation) should provide continuous interface between different pieces (fragments) of the surface and necessary aerodynamic properties. The task is to recover virtually (possibly approximately) the surface of fuselage using an optimal system of equations of lines that form the airframe, with the required technological and aerodynamic properties preserved.

The above is also true of the problem of describing surfaces of ships, rockets, automobiles, tailor dummies, etc.

Example 3. Radar Mapping of Planetary Surfaces. In mapping the surface of a space body using radar data sent from, for example, an artificial satellite (Fig. 3), it is necessary to use information on the equations of trajectories of a ray on the surface of a space body and on the surface geometry on these lines (or on adjacent strips).

Such a method is the only possible in some cases. For example, the surface of Venus cannot be seen through an optical telescope because of the thick layer of atmosphere. However, a map of its surface was created based on radar data. A surface mapping to be efficient, it should be supplemented with appropriate mathematics.

Example 4. Experiment Planning to Recover a Function of Two Variables. The following approach is often used in planning and conducting experiments. Let it be necessary to find a formula (probably, approximate) $z=f(x, y)$ describing a process that depends on two variables (parameters): $x$ and $y$. To this end, the values $x_{1}, \ldots, x_{m}$ of the parameter $x$ $\left(x=x_{k}, k=\overline{1, m}\right)$ are usually fixed, and functions $\psi_{k}(y)=f\left(x_{k}, y\right)$ for these values are found (the functions $\psi_{k}(y)$ can be represented as curves drawn by an automatic recorder or approximately, using their values at some points $\left.y_{1}<y_{2}<\ldots<y_{n}\right)$. Naturally, the variables $x$ and $y$ can be swapped: we fix values $y_{1}, \ldots, y_{n}$ of the parameter $y$ and find functions $\varphi_{l}(x)=f\left(x, y_{l}\right)(l=\overline{1, n})$ for $y=y_{l}$. The information obtained can be used to approximate the function $f(x, y)$ either by a Lagrange polynomial in the variable $x$ (or $y$ ), or by a spline that interpolates experimental functions $f\left(x_{k}, y_{l}\right)$ in the selected system of nodes $\left(x_{k}, y_{l}\right)$, or by a smoothing polynomial, a spline whose coefficients can be found by the least-squares method, etc. Note that either the values of $f\left(x_{k}, y_{l}\right)$, or the functions $\psi_{k}(y)(k=\overline{1, m})$, or $\varphi_{l}(x)(l=\overline{1, n})$ are often used in practice. Interlineations of functions allow simultaneous use of the values of the function $f\left(x_{k}, y_{l}\right)$ and the 


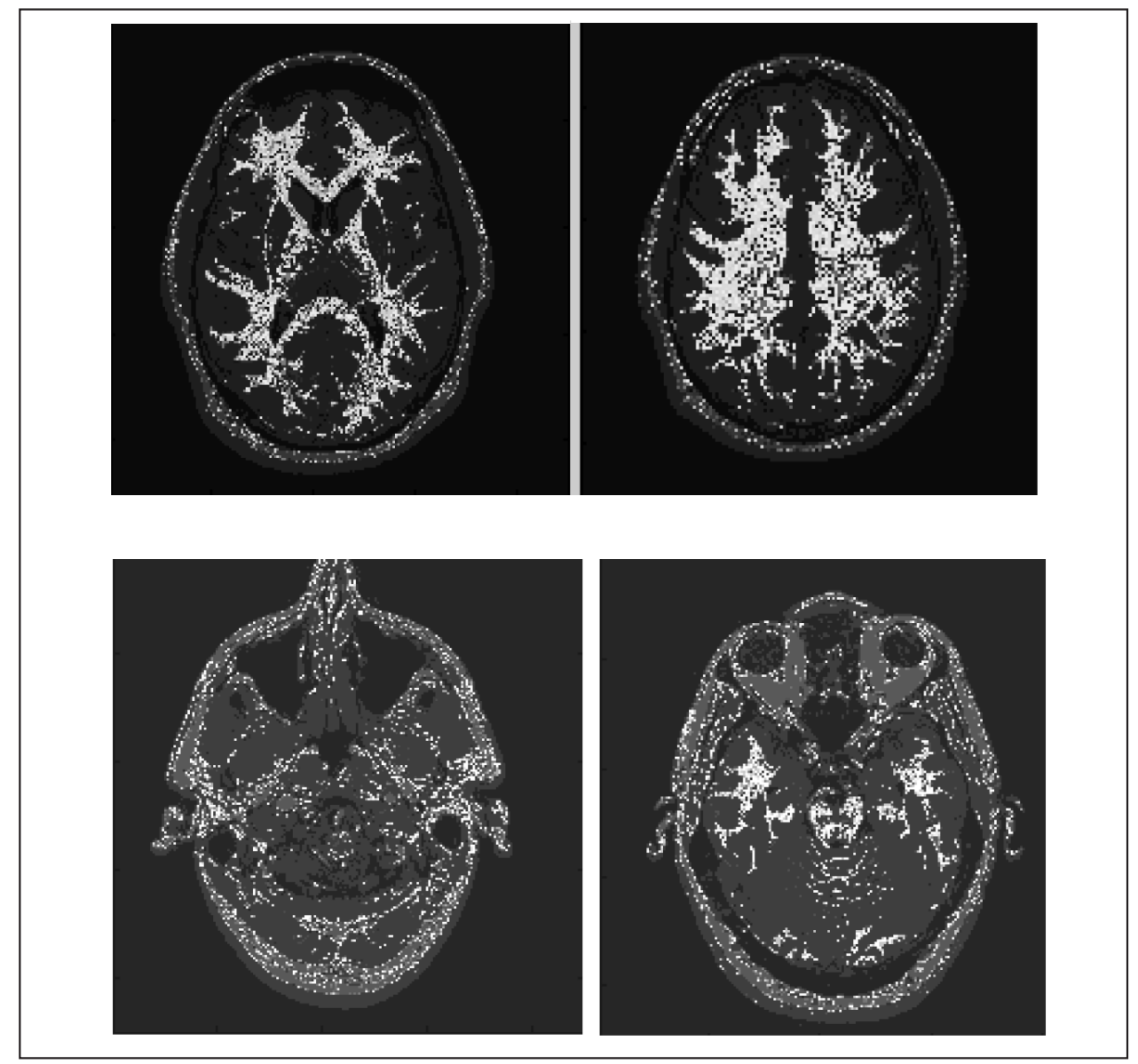

Fig. 4. Examples of images perpendicular to the $O Z$ axis.

functions $\psi_{k}(y)(k=\overline{1, m})$ and functions $\varphi_{l}(x)(l=\overline{1, n})$. Moreover, it becomes possible to efficiently process information given on a system of lines (not only straight lines parallel to the coordinate axes), which may considerably improve the accuracy of recovering the unknown function $f(x, y)$ and, what is especially important, to optimize the amount of data.

Example 5. Recovery of the Internal Structure of a Three-Dimensional Body Using Tomographic Images on Several Mutually Perpendicular Planes. Below we use the data on the internal structure of the brain provided by the Digital Anatomist package (100 images perpendicular to the $O X$ axis, 110 images perpendicular to the $O Y$ axis, and 35 images perpendicular to the $O Z$ axis). The intersection gap was $1.3 \mathrm{~mm}$ (Figs. 4-6).

How such information can be used to recover the internal structure of the body at any point? What would be the error? Section 3 presents the solution of this problem using operators of interflation of functions of three variables.

The science of the 21 st century poses a new variety of practically and theoretically important challenges, which have not yet been addressed in modern computational mathematics. Not only the initial information (experimental data) is nonconventional in these problems but also how it is acquired, stored, and presented for processing. Efficient solution of such problems requires new mathematical methods that would take into account not only the capabilities of modern computers but also the form of presentation of initial information (traces of functions, projections, images, tomograms, etc.).

In the present paper, we review the results from an analysis of computational methods that solve some of the above-mentioned problems with high accuracy. Therefore, the importance of the subject considered in the paper is obvious. The presentation is based on the theory of operators of interlineation and interflation that recover (possibly approximately) functions of $n(n \geq 2)$ variables using their traces and traces of their normal derivatives up to a given order $N$ on $M(M>1) m$-dimensional $(0 \leq m<n-1)$ sets in the $R^{n}$. 


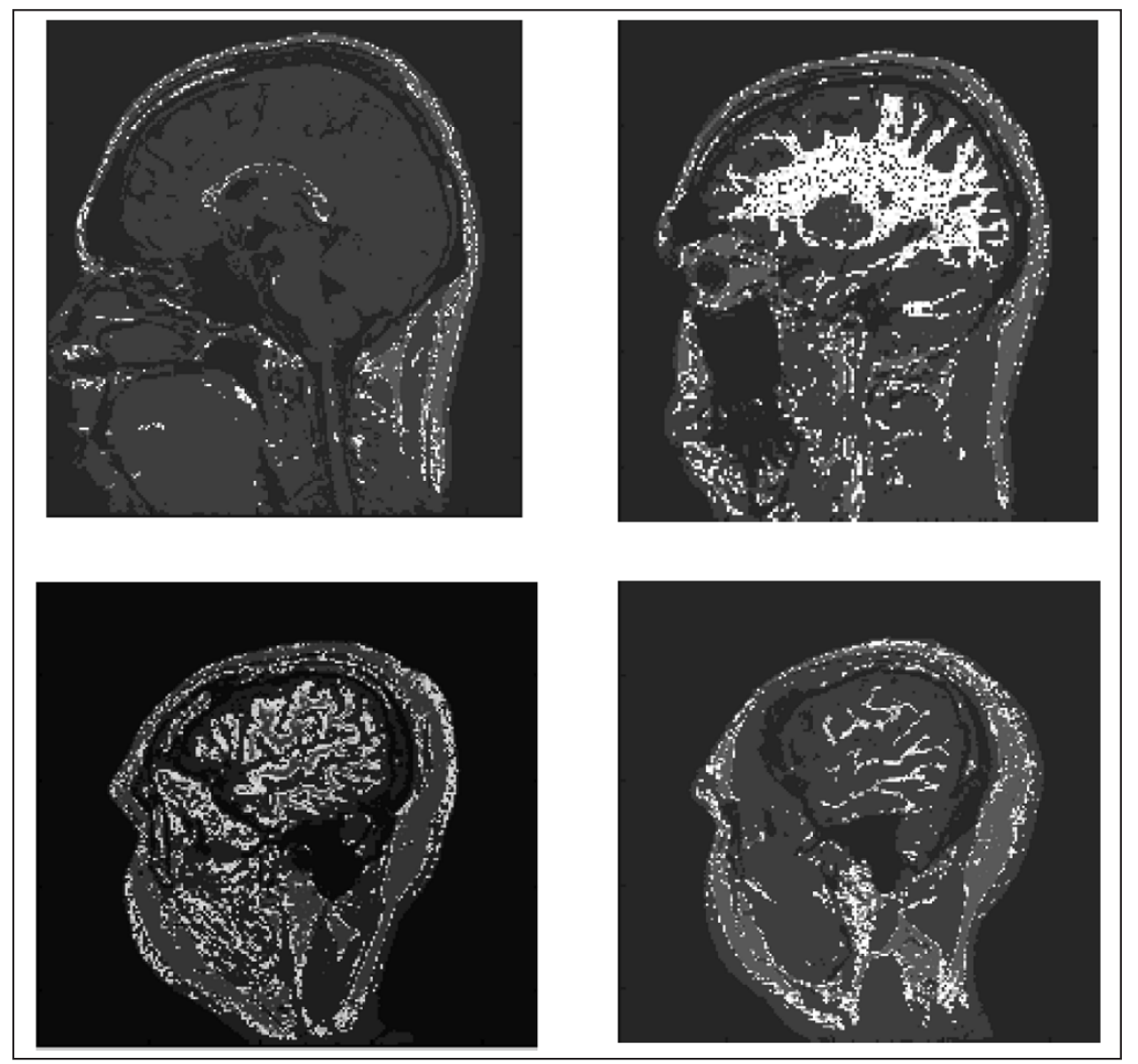

Fig. 5. Examples of images perpendicular to the $O Y$ axis.

\section{INTERLINEATION AND INTERFLATION OF FUNCTIONS. BASIC DEFINITIONS}

Let $n, M \in \mathrm{N}, m$, and $N \in \stackrel{0}{\mathrm{~N}}$ be given numbers, $\Pi_{k}, k=\overline{1, M}$, be given $m$-dimensional sets in the $R^{n}(0 \leq m<n)$; $\left.\varphi_{k, p}(x)\right|_{\Pi_{k}}=\left.L_{k, p} f(x)\right|_{\Pi_{k}}, k=\overline{1, M}, p=\overline{0, N}$, be given traces of the function $f(x)$ and traces of some operators $L_{k, p} f(x)$. The function $f$ can be unknown. The operators $L_{k, p} f(x)$ can be partial derivatives or normal derivatives: $\left.L_{k, p} f(x)\right|_{\Pi_{k}}=\partial^{p} f(x) /\left.\partial v_{k}^{p}\right|_{\Pi_{k}}, \quad p=\overline{0, N}$, for $m=n-1$, etc., $\quad\|\cdot\|=\|\cdot\|_{C}$.

Definition 1. We will call operators $O\left(\left\{\varphi_{k}, p\right\}, x\right):=O\left(\left\{L_{k, p}\right\},\left\{\Pi_{k}\right\},\left\{\varphi_{k, p}\right\}, x\right)$ the interflation operators if $\left.L_{l, q} O\left(\left\{\varphi_{k, p}\right\}, x\right)\right|_{\Pi_{l}}=\left.\varphi_{l, q}(x)\right|_{\Pi_{l}}, l=\overline{1, M}, q=\overline{0, N}$.

If $m=0$, then $O\left(\left\{\varphi_{k, p}\right\}, x\right)$ are the interpolation operators at $M$ points. If $m=1$ and $n \geq 2$, then $\Pi_{k}$ are lines in $R^{n}$ and $O\left(\left\{\varphi_{k, p}\right\}, x\right)$ are interlineation operators. Table 1 compares interpolation, interlineation, and interflation.

Since interlineation is a natural generalization of interpolation, we will analyze interlineation or interflation operators using the terminology from interpolation theory, replacing the "polation" part with "lineation" or "flation" without additional explanations.

Definition 2. Let $O\left(\left\{\varphi_{k, p}\right\}, x\right)=\sum_{l=1}^{M} \sum_{q=0}^{N} Y_{l, q}\left(\left\{\varphi_{k, p}\right\}, x\right) h_{l, q}(x)$, where $h_{l, q}(x)=h_{l, q}\left(\left\{L_{k, p}\right\},\left\{\Pi_{k}\right\}, x\right)$ are some auxiliary functions independent of the approximated function $f(x)$, and $\Upsilon_{l, q}\left(\left\{\varphi_{k, p}\right\}, x\right)=\Upsilon_{l, q}\left(\left\{L_{k, p}\right\},\left\{\Pi_{k}\right\},\left\{\varphi_{k, p}\right\}, x\right)$ are linear operators based on the functions $\varphi_{k, p}, k=\overline{1, M}, p=\overline{0, N}$. Then we will call $O\left(\left\{\varphi_{k, p}\right\}, x\right)$ linear-interflation operators. In the remaining cases, they are nonlinear-interflation operators. 


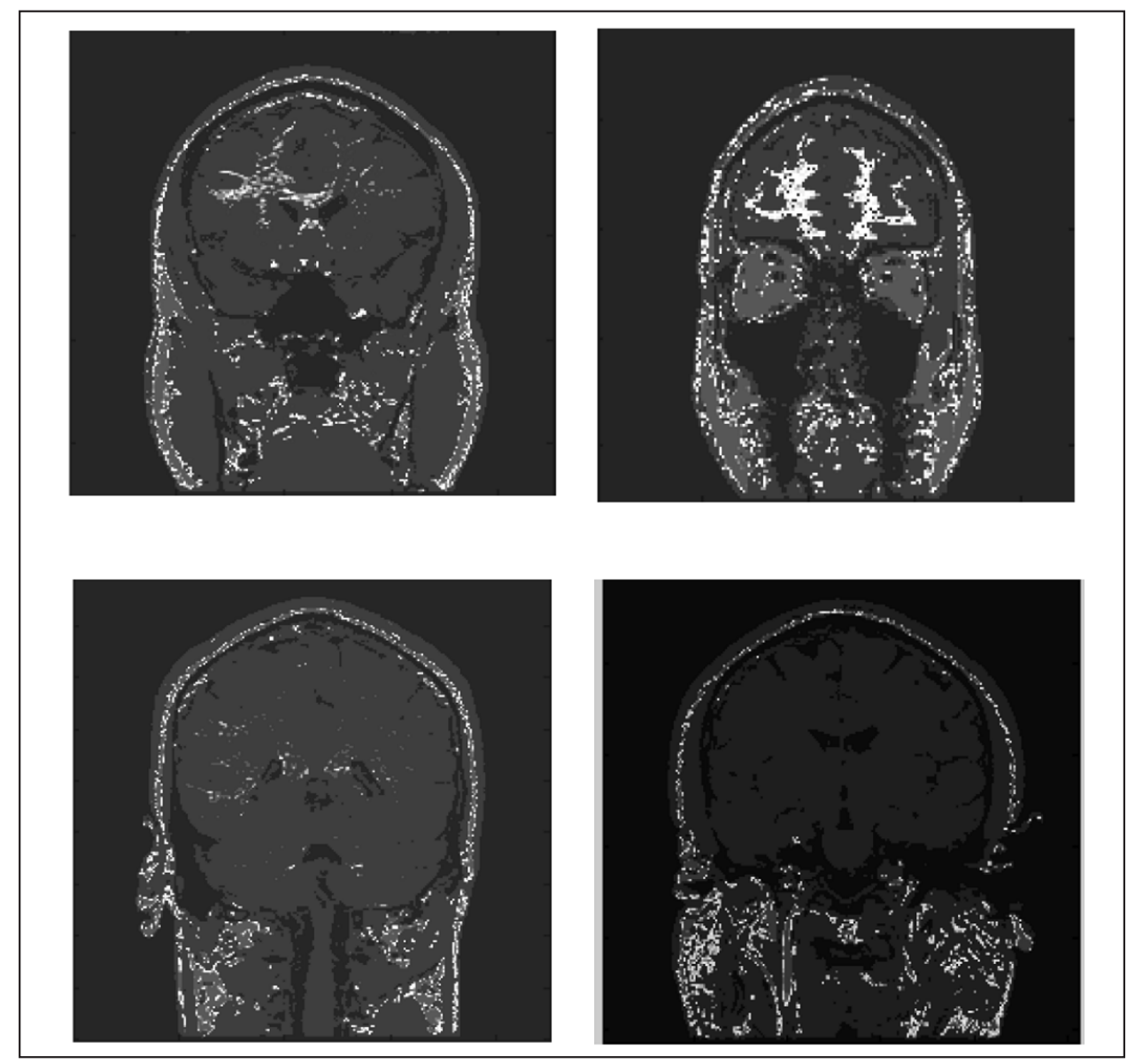

Fig. 6. Examples of images perpendicular to the $O X$ axis.

TABLE 1

\begin{tabular}{|l|l|}
\hline $\begin{array}{c}\text { Method of function } \\
\text { approximation }\end{array}$ & \multicolumn{1}{c|}{$\begin{array}{c}\text { Method of presenting } \\
\text { information }\end{array}$} \\
\hline $\begin{array}{l}\text { Interpolation of } \\
\text { functions of } n(n \geq 1) \\
\text { variables }\end{array}$ & $\begin{array}{l}\text { Values of the approximated } \\
\text { function and its derivatives } \\
\text { up to a fixed order in a } \\
\text { given system of points }\end{array}$ \\
\hline $\begin{array}{l}\text { Interlineation of } \\
\text { functions of } n(n \geq 2) \\
\text { variables (blending } \\
\text { function interpolation) }\end{array}$ & $\begin{array}{l}\text { Traces of the approximated } \\
\text { function and its normal } \\
\text { derivatives up to a fixed } \\
\text { order in a given system of } \\
\text { lines }\end{array}$ \\
\hline $\begin{array}{l}\text { Interflation of } \\
\text { functions of variables }\end{array}$ & $\begin{array}{l}\text { Traces of the approximated } \\
\text { function and its normal } \\
\text { derivatives up to a fixed } \\
\text { order on a given system of } \\
\text { surfaces }\end{array}$ \\
\hline
\end{tabular}

Definition 3. Let the auxiliary functions $h_{l, q}(x)=h_{l, q}\left(\left\{L_{k, p}\right\},\left\{\Pi_{k}\right\}, x\right)$ be rational, polynomial, trigonometric functions, spline functions or functions based on $R$-functions, etc. Then the operators $O\left(\left\{\varphi_{k}, p\right\}, x\right)$ are called operators of rational, polynomial, trigonometric or spline interflation, interflation based on $R$-functions, etc.

Definition 4. If $f(x) \in C^{r}\left(R^{n}\right), r \geq N \geq 1$, and $O\left(\left\{\varphi_{k}, p\right\}, x\right) \in C^{r}\left(R^{n}\right)$, then the operators $O\left(\left\{\varphi_{k}, p\right\}, x\right)$ are considered to preserve the differentiability class $C^{r}\left(R^{n}\right)$ to which the function $f(x)$ belongs. Otherwise, the operators $O\left(\left\{\varphi_{k, p}\right\}, x\right)$ do not preserve the differentiability class. 
Definition 5. If $\exists l, q:\left.L_{l, q} O\left(\left\{\varphi_{k, p}\right\}, x\right)\right|_{\Pi_{l}} \neq\left.\varphi_{l, q}(x)\right|_{\Pi_{l}}$, then $O\left(\left\{\varphi_{k, p}\right\}, x\right)$ are operators of mixed rational, polynomial, trigonometric, or spline approximation if the auxiliary functions $h_{l, q}(x)$ are rational, polynomial, trigonometric functions or spline functions, respectively.

\section{HISTORICAL ESSAY}

The monograph [18] provides a complete bibliography on this problem (till 2001). Therefore, we will mention only major early studies and new publications.

An interlineation problem arose every time when it was necessary to solve boundary-value problems with inhomogeneous boundary conditions at the interface of complex domains, whose boundary combines several lines. The general approach to explicit solutions of boundary-value problems involves integral representation of the solution in terms of its boundary values and the right-hand side of the differential equation. The Green, Kirchhoff, Poisson, and d'Alembert formulas and those from the theory of potentials, etc. are used. The main shortcoming of these formulas is that they cannot be constructively derived for complex domains.

The formulas for deriving operators of interlineation and interflation (blending function interpolation) of functions provide a different approach. A formula for the operator of polynomial interlineation on the sides of a rectangle was published first in [50] and then in $[47,1,2]$ for an arbitrary system of mutually perpendicular straight lines. The method of constructing operators of polynomial interlineation on a system of intersected straight lines lying in a plane, and interflation on a system of intersected planes in a space was first proposed in [19, 20]. V. L. Rvachev [44] offered a general method to construct interlineation operators based on $R$-functions. All the above-mentioned methods produce formulas that do not generally preserve the differentiability class $C^{r}\left(R^{2}\right)$ to which the approximated function belongs. The general method to construct interlineation operators preserving the differentiability class $C^{r}\left(R^{2}\right)$ to which the approximated function of two variables belongs was stated first in [18, Ch. 2; 23, Ch. 2]). The general method to construct economic operators of spline interpolation of functions of two variables (that require fewer values of the approximated function compared with classical splines of the same degree to achieve prescribed accuracy) based on spline interlineation operators is used in [46, 13] (see $[18,19]$ for economic spline interpolation of functions of three variables based on spline interflation). Some exact error estimates for functions of two variables approximated by a mixed sum of operators of one variable are obtained in [14]. Results on optimal polynomial and optimal trigonometric blending approximation of functions of two variables were first obtained in [48] and [49], respectively. Reducing boundary-value problems for partial differential equations to a system of ordinary linear integro-differential equations (LIDE) was analyzed in [17, 41-43]. A formula for operators of two-sided approximation of functions of two variables by using operators of polynomial interlineation on a system of mutually perpendicular straight lines was first proposed in [12]. New methods of solving plane and spatial problems of Radon tomography are analyzed in [3, 25, 26] and [4, 28-29], respectively. Error estimates for the Fourier coefficient approximated based on spline interlineation of functions of two variables and the Filon method are analyzed in [24]. Methods for computing Fourier coefficients and Hartley functions of two and three variables are developed in [30-40]. These methods are based on spline interlineation and spline interflation of functions together with the Filon method, trend analysis, efficient construction of Fourier and Hartley sums, which are exact on trigonometric polynomials of prescribed order, despite the fact that approximate cubature formulas are used to compute Fourier and Hartley coefficients. To develop auxiliary functions in the formulas of spline interlineation and spline interflation, splines of various orders are used: piecewise constant, of the first, third, and fifth orders of interpolation and approximation types. A method of solving a nonstationary heat-conduction problem using interlineation and interflation of functions was studied first in [45, 11]. The application of interlineation and interflation of functions in digital processing of multidimensional signals is analyzed in [22].

If the domain of an approximation operator consists of several domains and its boundary consists of several lines (or surfaces), then designing functions with prescribed boundary conditions is a key problem in finding approximate solutions to boundary-value problems. In the second half of the 20th century, V. L. Rvachev and his disciples developed a general algorithm based on $R$-functions to satisfy boundary conditions that include partial differential operators up to a fixed order $N$. However, functions designed using such an algorithm may not belong to the same differentiability class to which the approximated function belongs (for $N \geq 1$ ). Operators that exactly satisfy boundary conditions and preserve (or do not preserve) the differentiability class of the approximated function can be constructed using corresponding operators of interlineation and interflation [5-10]. 


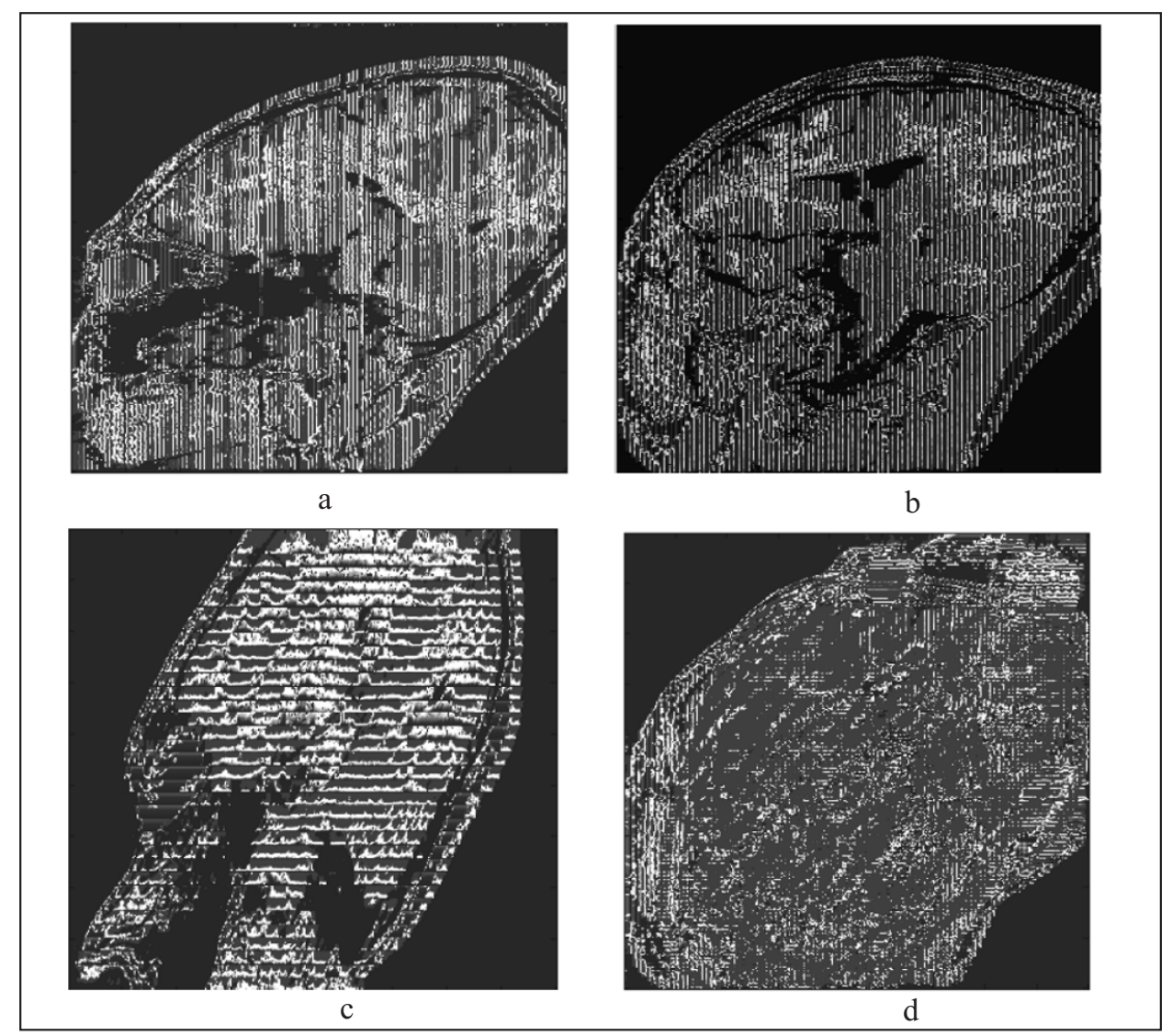

Fig. 7. Brain structure in the plane $x+y+10 z+1=0$ using Brainox (a), Brainoy (b), Brainoz (c), and Brain (d) software.

All the example problems above can efficiently be solved using interlineation and interflation of functions. We present below only the solution to the problem from Example 5 using the software created by the authors based on spline interflation of functions $[28,29]$. Figure 7 presents approximate recovery of the internal structure of the brain in the plane $x+y+10 z+1=0$. Four methods were compared. The first three methods (Brainox, Brainoy, and Brainoz) used the information contained in each of the three series of images (perpendicular to one coordinate axis). The fourth method (Brain) proposed in $[28,29]$ used all the three systems of images obtained in different, mutually perpendicular planes.

The section in Fig. 7d suggests that the use of all the data in all the three mutually perpendicular planes produces a higher accuracy in the considered section than the use of the data parallel to one plane (perpendicular to one axis) alone. Economic operators of mixed wavelet approximation of functions of two variables based on Haar wavelets are analyzed in $[15,16]$. The methods of optimizing the number of experimental data to recover the surface of a three-dimensional body are studied in [27]. The methods considered are applied to optimize the surface of a tailor dummy.

Figure 8 shows the surface of a tailor dummy constructed from experimental data and fewer horizontal $(m)$ and vertical $(n)$ lines on its surface, preserving a prescribed accuracy.

\section{INTERLINEATION OPERATORS WITHOUT PRESERVING THE CLASS $C^{r}\left(R^{2}\right), r \geq 1$}

1. Operators of Rational Interlineation on $\boldsymbol{M}$ Lines. Let $n=2$ and $\Pi_{k}: \omega_{k}(x):=a_{k} x_{1}+b_{k} x_{2}-\gamma_{k}=0, \quad k=\overline{1, M}$, $a_{k}^{2}+b_{k}^{2}=1$

$$
\begin{aligned}
\varphi_{k, s}(x)=\partial^{s} f /\left.\partial v_{k}^{s}(x)\right|_{\Pi_{k}} & =\partial^{s} f / \partial v_{k}^{s}\left(x_{1},\left(\gamma_{k}-a_{k} x_{1}\right) / b_{k}\right) \text { if } b_{k} \neq 0 ; \\
v_{k} & =\nabla \omega_{k}(x)=\left(a_{k}, b_{k}\right)
\end{aligned}
$$




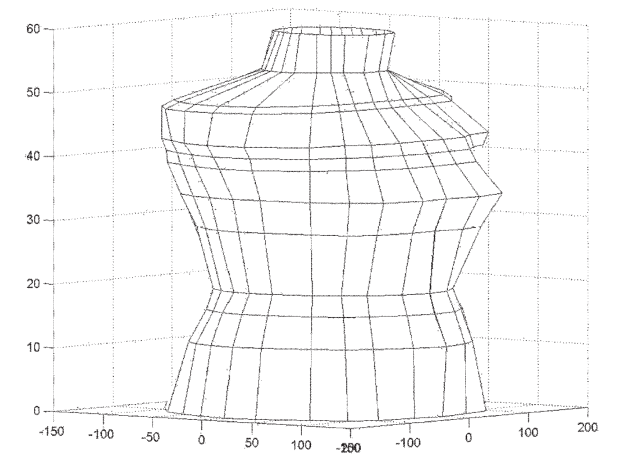

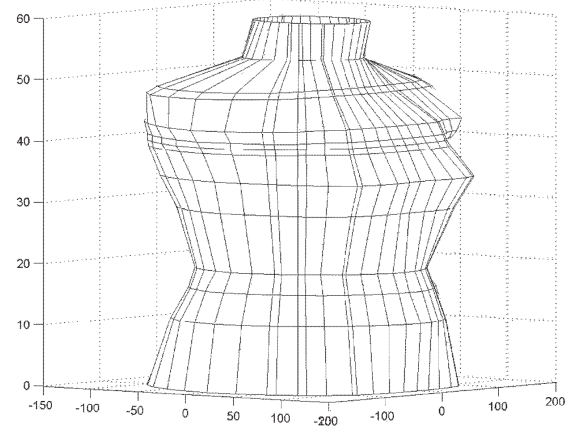

b

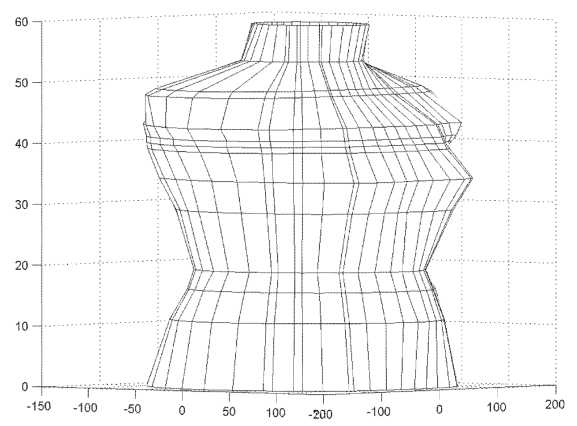

c

Fig. 8. Approximate surface of a tailor dummy: (a) for $m=13, n=13$, (b) for $m=14, n=13$, (c) the original model.

$$
\begin{gathered}
x-\omega_{k}(x) \nabla \omega_{k}(x)=\left(x_{1}-\omega_{k}(x) \frac{\partial \omega_{k}(x)}{\partial x_{1}}, \quad x_{2}-\omega_{k}(x) \frac{\partial \omega_{k}(x)}{\partial x_{2}}\right) \\
\text { or } \varphi_{k, s}(x)=\partial^{s} f /\left.\partial v_{k}^{s}(x)\right|_{\Pi_{k}}=\partial^{s} f / \partial v_{k}^{s}\left(\left(\gamma_{k}-b_{k} x_{2}\right) / a_{k}, x_{2}\right) \text {, if } a_{k} \neq 0, \\
O_{k, N} f(x)=\sum_{s=0}^{N} \varphi_{k, s}\left(x-\omega_{k}(x) \nabla \omega_{k}(x)\right) \frac{\omega_{k}^{s}(x)}{s !}, \quad H_{k}(x)=\prod_{\substack{i=1 \\
i \neq k}}^{M} \omega_{i}^{N^{*}}(x) / \sum_{l=1}^{M} \prod_{\substack{i=1 \\
i \neq l}}^{M} \omega_{i}^{N^{*}}(x) ; \\
N^{*}=N+1, \text { if } N=2 q+1, q \in \stackrel{0}{\mathrm{~N}} ; N^{*}=N+2 \text { if } N=2 q, q \in \stackrel{N}{N} .
\end{gathered}
$$

THEOREM 1. The operator $O_{M, N}\left(\left\{\varphi_{k, s}\right\},\left\{\Pi_{k}\right\}, x\right)=\sum_{k=1}^{M} O_{k, N} f(x) H_{k, N}(x)$ has the following properties: $\partial^{s} O_{M, N}\left(\left\{\varphi_{k, s}\right\},\left\{\Pi_{k}\right\}, x\right) /\left.\partial v_{k}^{s}(x)\right|_{\Pi_{k}}=\left.\varphi_{k, s}(x)\right|_{\Pi_{k}}, k=\overline{1, M}, \quad s=\overline{0, N}$.

If $f(x) \in C^{r}\left(R^{2}\right), r \geq N+1$, and $\left.\partial^{s} f(x)\right|_{\Pi_{k}}=\left.\varphi_{k, s}(x)\right|_{\Pi_{k}}, k=\overline{1, M}, s=\overline{0, N}$, then the residue $R_{N} f(x)=(I-$ $\left.O_{M, N}\left(\left\{\varphi_{k, s}\right\},\left\{\Pi_{k}\right\}, x\right)\right)$ satisfies the relation $R_{N} f(x)=O\left(\prod_{k=1}^{M} \omega_{k}^{N+1}(x)\right)$ if $\prod_{k=1}^{M} \omega_{k}^{N+1}(x) \rightarrow 0$.

Remark 1. If $\Pi_{k}: \omega_{k}(x)=0, k=\overline{1, M}$, is an arbitrary set of lines or surfaces in $R^{n}, n \geq 2$, and $\partial^{p} \omega_{k}(x) /\left.\partial v_{k}^{p}\right|_{\Pi_{k}}$ $=\delta_{0, p}, \quad p=\overline{1, N^{*}}, N^{*}>N$, then the statement of Theorem 1 remains in force. 
2. Polynomial, Trigonometric or Spline Interlineation on a Set of Mutually Perpendicular Straight Lines. Let $G=I^{2}, I=[0,1], 0=x_{k, 0}<\ldots<x_{k, M_{k}}=1, k=1,2 ; \partial^{s_{k}} f(x) /\left.\partial x_{k}^{s_{k}}\right|_{x_{k}}=x_{k, i_{k}}=\varphi_{k, i_{k}, s_{k}}\left(x_{3-k}\right)$,

$$
B_{k} f(x)=\sum_{i_{k}=0}^{M_{k}} \sum_{s_{k}=0}^{N} \varphi_{i_{k}, s_{k}}\left(x_{3-k}\right) h_{M_{k}, N, s_{k}}\left(x_{k}\right), h_{M_{k}, i_{k}, s_{k}}^{(q)}\left(x_{k, j}\right)=\delta_{q, i_{k}} \delta_{i_{k}, j}, \quad q, s_{k}=\overline{0, N} ; i_{k}, j=\overline{1, M_{k}},
$$

$h_{M_{k}, N, s_{k}}\left(x_{k}\right)$ being a system of basis functions of polynomial, trigonometric or spline interpolation. The operators $B_{k} f(x)$ act on one variable $x_{k}(k=1,2)$. Then $L f(x)=B_{1} B_{2} f(x)$ are the classical operators of polynomial, trigonometric or spline interpolation on a set of points $\left(x_{1, i}, x_{2, j}\right), i=\overline{1, N_{1}}, j=\overline{1, N_{2}}$.

THEOREM 2. The operator $O f(x)=\left(B_{1}+B_{2}-B_{1} B_{2}\right) f(x)$ has the following properties:

$$
\begin{gathered}
\partial^{p} O f(x) / \partial x_{k}^{p}=\partial^{p} f(x) / \partial x_{k}^{p}=\varphi_{k, i_{k}, s_{k}}\left(x_{3-k}\right), \\
x_{k}=x_{k, i_{k}}, \quad p=\overline{0, N}, i_{k}=\overline{0, M_{k}}, k=1,2, \quad R_{12} f(x):=(I-O) f(x)=\left(I-B_{1}\right)\left(I-B_{2}\right) f(x) .
\end{gathered}
$$

In other words, $R_{12} f(x)=O\left(\varepsilon^{2}\right)$ if $\left(I-B_{k}\right) f(x)=O(\varepsilon), \varepsilon \rightarrow 0, k=1,2$. A polynomial case on an arbitrary system of straight lines $(n=2)$ and planes $(n=3)$ is considered in [18].

3. Economic Operators of Polynomial, Trigonometric and Spline Interpolation Based on Interlineation Operators. In the general case, these operators have the form $\bar{O} f(x)=\left(\bar{B}_{1}+\bar{B}_{2}-B_{1} B_{2}\right) f(x)$. The operators $\bar{B}_{k} f(x)$ can be obtained by substituting $\Phi_{i_{k}, s_{k}}\left(x_{3-k}\right) \approx \varphi_{i_{k}, s_{k}}\left(x_{3-k}\right)$ into the operators $B_{k} f(x)$, where $\Phi_{i_{k}, s_{k}}\left(x_{3-k}\right)$ are operators of polynomial, trigonometric or spline interpolation that act on functions of one variable and have the properties $\left\|\varphi_{i_{k}, s_{k}}\left(x_{3-k}\right)-\Phi_{i_{k}, s_{k}}\left(x_{3-k}\right)\right\|=o\left(\varepsilon^{2}\right)$.

THEOREM 3. The interpolation operator $\bar{O} f(x)$ employs fewer values of the function $f(x)$ than the interpolation operator $B_{1} B_{2} f(x)$ does (if both of them approximate the function $f(x)$ with an error $O\left(\varepsilon^{2}\right)$ ).

\section{OPERATORS OF INTERLINEATION AND INTERFLATION PRESERVING THE CLASS $C^{r}\left(R^{n}\right), r \geq 1$}

If $N \geq 1$, then it is recommended to use the operators that preserve the differentiability class $C^{r}\left(R^{n}\right), r \geq 1$, to which the approximated function belongs. They are constructed using solutions to some partial differential equations.

1. Discrete Generalized D'Alembert Formula.

THEOREM 4. Let

$$
\begin{gathered}
f\left(x_{1}, x_{2}\right) \in C^{r}\left(R^{2}\right), r>N \geq 0, f^{(p, s)}(x)=: \partial^{P+s} f / \partial x_{1}^{p} \partial x_{2}^{s} ; \\
f^{(0, s)}\left(x_{1}, 0\right)=\varphi_{s}\left(x_{1}\right) \in C^{r-s}\left(R^{1}\right), s=\overline{0, N},
\end{gathered}
$$

and $\beta_{0}, \beta_{1}, \ldots, \beta_{N}$ be given different (or multiple) real (or complex) numbers. Then the operator

$$
D_{N} f(x)=\sum_{i=0}^{N} \lambda_{N o i} f\left(x_{1}+\beta_{i} x_{2}, 0\right)+\sum_{s=1}^{N} \sum_{i=0}^{N} \lambda_{N s i} \int_{0}^{x_{1}+\beta_{i} x_{2}} f^{(0, s)}(t, 0) \frac{\left(x_{1}+\beta_{i} x_{2}-t\right)^{s-1}}{(s-1) !} d t,
$$

where $\lambda_{N s i}, 0 \leq i, s \leq N$, is a solution of the systems

$$
\sum_{i=0}^{T} \lambda_{N s i} \beta_{i}^{p}=\delta_{p, s}, 0 \leq p, s \leq N
$$

has the following properties: $D_{N} f(x) \in C^{r}\left(R^{2}\right)$,

$$
\left.\frac{\partial^{p} D_{N} f(x)}{\partial x_{2}^{p}}\right|_{x_{2}=0}=f^{(0, s)}\left(x_{1}, 0\right)=\varphi_{s}\left(x_{1}\right), s=\overline{0, N}
$$


If $R_{N} f(x)=\left(I-D_{N}\right) f(x)$, then

$$
\begin{gathered}
R_{N} f(x)=\int_{0}^{x_{2}}\left[\sum_{i=0}^{N} \Delta_{N i}^{-1} \int_{0}^{x_{1}+\beta_{i}\left(x_{2}-z\right)} A_{N+1} f(t, z) \frac{\left(x_{1}+\beta_{i}\left(x_{2}-z\right)-t\right)^{N-1}}{(N-1) !} d t\right] d z, \\
A_{N+1} f(x)=: \prod_{v=0}^{N}\left(-\beta_{v} \frac{\partial}{\partial x_{1}}+\frac{\partial}{\partial x_{2}}\right) f(x), x=\left(x_{1}, x_{2}\right), \Delta_{N i}=\prod_{\substack{v=0 \\
v \neq i}}^{N}\left(\beta_{i}-\beta_{v}\right), \\
M_{N+1}=R_{N} f(x) \mid \leq M_{N+1} h^{N+1} /(N+1) !, \\
\max _{t \geq 0,0 \leq z \leq x_{2} \leq h}\left|A_{N+1} f(t, z)\right|=: M_{N+1}\left(\beta_{0}, \ldots, \beta_{N}\right) .
\end{gathered}
$$

In other words, the operators $D_{N} f$ preserve the differentiability class of the function $f$.

COROLLARY. There exists a unique solution of the problem

$$
A_{N+1} f(x)=g(x), g(x) \in C\left(R^{2}\right), f^{(0, s)}\left(x_{1}, 0\right)=\varphi_{s}\left(x_{1}\right) \in C^{N+1-s}\left(R^{1}\right), s=\overline{0, N},
$$

in the form

$$
f(x)=D_{N} f(x)+\int_{0}^{x_{2}}\left[\sum_{i=0}^{N} \Delta_{N i}^{-1} \int_{0}^{x_{1}+\beta_{i}\left(x_{2}-z\right)} g(t, z) \frac{\left(x_{1}+\beta_{i}\left(x_{2}-z\right)-t\right)^{N-1}}{(N-1) !} d t\right] d z .
$$

THEOREM 5. Let $x_{2 k}, k=\overline{1, M}, \beta_{i}, i=\overline{0, N}$, be given different numbers,

$$
\begin{gathered}
h_{k, s}\left(x_{2}\right)=\prod_{\substack{\nu=1 \\
v \neq k}}^{M}\left(x_{2}-x_{2 k}\right)^{N+1}\left\{\prod_{\substack{\nu=1 \\
v \neq k}}^{M}\left(x_{2}-x_{2 k}\right)^{-N-1}\right\}_{\left(x_{2 k}\right)}^{(N-s)}, \\
\{g(t)\}_{(u)}^{(q)}:=\sum_{m=0}^{q} g^{(m)}(u) \frac{(t-u)^{m}}{m !} .
\end{gathered}
$$

Then the operators

$$
\begin{gathered}
E_{M, N, \beta} f(x)=\sum_{k=1}^{M} h_{k, 0}\left(x_{2}\right) \sum_{i=0}^{N} \lambda_{N, 0, i} f\left(x_{1}+\beta_{i}\left(x_{2}-x_{2 k}\right), x_{2 k}\right) \\
+\sum_{k=1}^{M} \sum_{s=1}^{N} h_{k, s}\left(x_{2}\right) \sum_{i=0}^{N} \lambda_{N s i} \int_{x_{2 k}}^{x_{1}+\beta_{i}\left(x_{2}-x_{2 k}\right)} f^{(0, s)}\left(t, x_{2 k}\right) \frac{\left(x_{1}+\beta_{i}\left(x_{2}-x_{2 k}\right)-t\right)^{s-1}}{(s-1) !} d t
\end{gathered}
$$

have the following properties:

$$
\begin{gathered}
f(x) \in C^{r}\left(R^{2}\right) \Rightarrow E_{M, N, \beta} f(x) \in C^{r}\left(R^{2}\right), r \geq N, \beta=\left\{\beta_{i}\right\}, \\
\left.\frac{\partial^{p} E_{M, N, \beta} f(x)}{\partial x_{2}^{p}}\right|_{x_{2}=x_{2 k}}=f^{(o, p)}\left(x_{1}, x_{2 k}\right), k=\overline{1, M}, p=\overline{0, N} .
\end{gathered}
$$

If $h_{k, s}\left(x_{2}\right)=h_{k, 0}\left(x_{2}\right), s=\overline{0, N}$, then

$\left(I-E_{M, N, \beta}\right) f(x)=\sum_{k=1}^{M} h_{k, 0}\left(x_{2}\right) \int_{x_{2 k}}^{x_{2}} \sum_{i=0}^{N} \Delta_{N, i}^{-1} \int_{0}^{x_{1}+\beta_{i}\left(x_{2}-x_{2 k}-z_{k}\right)}\left(A_{N+1} f\right)\left(t, z_{k}\right) \frac{\left(x_{1}+\beta_{i}\left(x_{2}-x_{2 k}-z_{k}\right)-t\right)^{N-1}}{(N-1) !} d t d z_{k}$. 
The statements of Theorem 5 can be generalized to the case of several straight lines in the $R^{2}$.

Let, as above,

$$
\begin{gathered}
\Gamma_{k}: \omega_{k}(x)=: a_{k} x_{1}+b_{k} x_{2}-c_{k}=0, a_{k}^{2}+b_{k}^{2}=1, v_{k}=\left(a_{k}, b_{k}\right)=\nabla \omega_{k} \perp \Gamma_{k}, \\
t_{k}=t_{k}(x)=: b_{k} x_{1}-a_{k} x_{2}, \tau_{k}=\nabla t_{k}=\left(b_{k},-a_{k}\right), \tau_{k}|| \Gamma_{k}, \\
f(x) \in C^{r}\left(R^{2}\right), r>N,\left.\frac{\partial^{s} f(x)}{\partial v_{k}^{s}}\right|_{\Gamma_{k}}=\left.f_{k, s}(x)\right|_{\Gamma_{k}}, k=\overline{1, M}, s=\overline{0, N}, \\
\Phi_{k}\left(t_{k}, \omega_{k}\right)=: f\left(t_{k} b_{k}+\omega_{k} a_{k}-c_{k} a_{k},-t_{k} a_{k}+\omega_{k} b_{k}-c_{k} b_{k}\right) \equiv f(x), \\
\left.\frac{\partial^{s} f(x)}{\partial v_{k}^{s}}\right|_{\Gamma_{k}}=\left.\frac{\partial^{s} \Phi_{k}\left(t_{k}, \omega_{k}\right)}{\partial v_{k}^{s}}\right|_{\omega_{k}=0}=\Phi_{k}^{(0, s)}\left(t_{k}, 0\right), k=\overline{1, M}, s=\overline{0, N},
\end{gathered}
$$

$\beta_{j}, j=\overline{0, N}$, being given numbers.

$$
\begin{aligned}
& D_{N, k} f(x)=\sum_{j=0}^{N} \lambda_{N, 0, j} \Phi_{k}\left(t_{k}+\beta_{j} \omega_{k}\right) \\
& +\sum_{s=1}^{N} \sum_{j=0}^{N} \lambda_{N, s, j} \int_{0}^{t_{k}+\beta_{j} \omega_{k}} \Phi_{k}^{(0, s)}\left(\xi_{k}, 0\right) \frac{\left(t_{k}+\beta_{j} \omega_{k}-\xi_{k}\right)^{s-1}}{(s-1) !} d \xi_{k}, \quad \bar{N}=\left\{\begin{array}{l}
N+1 \text { if } N=2 N^{*}, \\
N \quad \text { if } N=2 N^{*}+1,
\end{array}\right. \\
& \omega(x)=\prod_{i=1}^{M} \omega_{i}(x), \Omega_{M, N}(x)=\sum_{i=1}^{M} \omega^{\bar{N}+1}(x) \omega_{i}^{-\bar{N}-1}(x), \\
& h_{M, N, k}(x)=\frac{\omega^{\bar{N}+1}(x) \omega_{k}^{-\bar{N}-1}(x)}{\Omega_{M, N}(x)}, k=\overline{1, M} .
\end{aligned}
$$

THEOREM 6. The operators

$$
\widetilde{D}_{M, N} f(x)=\sum_{k=1}^{M} h_{M, N, k}(x) D_{N, k} f(x)
$$

have the following properties:

$$
\begin{gathered}
f(x) \in C^{r}\left(R^{2}\right) \Rightarrow \widetilde{D}_{M, N} f(x) \in C^{r}\left(R^{2} \backslash G\right), \quad r \geq N, G=\left\{\Gamma_{k} \cap \Gamma_{j}\right\}=\left\{A_{k_{j}}\right\}, \\
\left.\frac{\partial^{p} \widetilde{D}_{M, N} f(x)}{\partial v_{k}^{p}}\right|_{\Gamma_{k}}=\left.\frac{\partial^{p} f(x)}{\partial v_{k}^{p}}\right|_{\Gamma_{k}}, \quad p=\overline{0, N}, \quad k=\overline{1, M}, \\
\left(I-\widetilde{D}_{M, N}\right) f(x)=\sum_{k=1}^{M} h_{M, N, k}(x)\left(I-\widetilde{D}_{N, k}\right) f(x) .
\end{gathered}
$$

2. A Continuous Analog of the Generalized D'Alembert Formula. The formulas for $D_{N} f$ and $E_{M, N, \beta} f$ are called the discrete generalized d'Alembert formulas. Let us write them in an integral form. Let

$$
\begin{gathered}
\zeta(\beta) \in C^{\infty}\left(R^{1}\right), \xi(\beta)=0 \text { if } \beta \leq-1, \beta \geq 1, \int_{-\infty}^{\infty} \zeta(\beta) d \beta=1, \\
K_{N, s}(\beta)=\sum_{k=1}^{N+1} \Lambda_{N s k} \xi\left(\frac{\beta}{k}\right), \quad \int_{-\infty}^{\infty} K_{N, s}(\beta) \beta^{i} d \beta=\delta_{s, i}, \quad 0 \leq s, i \leq N .
\end{gathered}
$$

60 
The operators $\widetilde{D}_{N} f(x)$ below are continuous analogs of the integral operators $D_{N} f(x)$.

THEOREM 7. The operators

$$
\widetilde{D}_{N} f(x)=\int_{-\infty}^{\infty} K_{N, 0}(\beta) f\left(x_{1}+\beta x_{2}, 0\right) d \beta+\sum_{s=1}^{N} \int_{-\infty}^{\infty} K_{N, s}(\beta) \int_{x_{1}}^{x_{1}+\beta x_{2}} f^{(0, s)}(t, 0) \frac{\left(x_{1}+\beta x_{2}-t\right)^{s-1}}{(s-1) !} d t d \beta
$$

have the following properties: $f(x) \in C^{r}\left(R^{2}\right) \Rightarrow \widetilde{D}_{N} f(x) \in C^{r}\left(R^{2}\right), r \geq N$,

$$
\begin{gathered}
\left.\frac{\partial^{p} D_{N} f(x)}{\partial x_{2}^{p}}\right|_{x_{2}=0}=f^{(o, p)}\left(x_{1}, 0\right), p=\overline{0, N}, \\
\left(I-\widetilde{D}_{N}\right) f(x)=\int_{0}^{x_{2}}\left\{\int_{-\infty}^{\infty}\left[\int_{x_{1}}^{x_{1}+\beta\left(x_{2}-z\right)} \widetilde{A}_{N+1}(f ; t, z, \beta) \frac{\left(x_{1}+\beta\left(x_{2}-z\right)-t\right)^{N-1}}{(N-1) !} d t\right] d \beta\right\} d z, \\
\widetilde{A}_{N+1}(f ; t, z, \beta)=\left[K_{N, 0}(\beta) f^{(N+1,0)}+\sum_{s=1}^{N}\left(K_{N, s-1}(\beta)-\beta K_{N, s}(\beta)\right) f^{(N+1-s, s)}+K_{N, N}(\beta) f^{(0, N)}\right](t, z) .
\end{gathered}
$$

Thus, the operators $D_{N} f(x)$ and $\widetilde{D}_{N} f(x)$ recover differentiable functions so that not only traces on the given lines $\Gamma_{k}$ are preserved but also the differentiability class to which the approximated function $f(x)$ belongs (in contrast to the operators in Sec. 4, item 1).

These results can be generalized to the case $x=\left(x_{1}, \ldots, x_{n}\right), n>2$.

\section{ECONOMIC 3D INTERPOLATION OPERATORS BASED ON 3D INTERFLATION OPERATORS}

Let $f(x) \in C^{r, r, r}\left(I^{3}\right), r=1,2, h(t)=(|t-1|-2|t|+|t+1|) / 2, E=[0,1], \quad u_{k, i_{k}}(x)=\left.f(x)\right|_{x_{k}=i_{k} / M}, 0 \leq i_{k} \leq M, k=\overline{1,3}$,

$$
L_{k, M} f(x)=\sum_{i_{k}=0}^{M} u_{k, i_{k}}(x) h\left(M x_{k}-i_{k}\right)
$$

THEOREM 8. The operator $O f(x)=\left(L_{1, M}+L_{2, M}+L_{3, M}-L_{1, M} L_{2, M}-L_{1, M} L_{3, M}-L_{2, M} L_{3, M}+\right.$ $\left.L_{1, M} L_{2}, M L_{3, M}\right) f(x)$ has the following properties:

$$
\begin{gathered}
\left.O f(x)\right|_{x_{k}=j_{k} / M}=\left.f(x)\right|_{x_{k}=j_{k} / M}, j_{k}=\overline{0, M}, k=\overline{1,3}, \\
\| f-O f||=O\left(M^{-3 r}\right) \quad \forall u \in C^{r, r, r}\left(I^{3}\right), r=1,2 .
\end{gathered}
$$

THEOREM 9. Let

$$
\begin{aligned}
u_{1, i_{1}}(x)=f\left(i_{1} / M, x_{2}, x_{3}\right) \approx \widetilde{u}_{1, i_{1}}(x)=\sum_{j_{2}=0}^{M^{3 / 2}} \sum_{j_{3}=0}^{M^{3}} f\left(i_{1} / M, j_{2} / M^{3 / 2}, j_{3} / M^{3}\right) h\left(M^{3 / 2} x_{2}-j_{2}\right) h\left(M^{3} x_{3}-j_{3}\right) \\
\quad+\sum_{j_{2}=0}^{M^{3}} \sum_{j_{3}=0}^{M^{3 / 2}} f\left(i_{1} / M, j_{2} / M^{3}, j_{3} / M^{3 / 2}\right) h\left(M^{3} x_{2}-j_{2}\right) h\left(M^{3 / 2} x_{3}-j_{3}\right) \\
\quad-\sum_{j_{2}=0}^{M^{3 / 2}} \sum_{j_{3}=0}^{M^{3 / 2}} f\left(i_{1} / M, j_{2} / M^{3 / 2}, j_{3} / M^{3 / 2}\right) h\left(M^{3 / 2} x_{2}-j_{2}\right) h\left(M^{3 / 2} x_{3}-j_{3}\right) .
\end{aligned}
$$


We will also make similar substitutions for the remaining two- and one-variable functions in $O f$ to obtain the operator $\bar{O} f(x)$ with the following properties:

(1) $\|f-\bar{O} f\|=O\left(M^{-3 r}\right)$;

(2) the operator $\bar{O} f(x)$ uses $Q=6(M+1)\left(M^{3 / 2}+1\right)\left(M^{3}+1\right)=O\left(M^{5.5}\right)$ values of the function $f$. Note that classical operators of three-linear interpolation (linear in each of the three variables) $L_{1, M^{3}} L_{2, M^{3}} L_{3, M^{3}} f(x)$ have an error $\left\|f-L_{1, M^{3}} L_{2, M^{3}} L_{3, M^{3}} f\right\|=O\left(M^{-3 r}\right)$ of the same order but employ $Q_{\text {classic }}=\left(M^{3}+1\right)^{3}=O\left(M^{9}\right)$ values of the function $f$. The statement of Theorem 9 is also true when splines of higher order or piecewise constant splines are used.

7. Approximation Operators Based on Operators of Mixed Approximation of Functions. Let $f(x) \in C^{r}(G)$, $x=\left(x_{1}, x_{2}\right), B_{k, N} f(x), k=1,2 ; N \geq 2$, be operators of one-dimensional approximation that act on the variable $x_{k}$, and the other variable be considered as a parameter. Then $B_{N} f(x)=\left(B_{1, N}+B_{2, N}-B_{1, N} B_{2, N}\right) f(x)$ is an operator of mixed approximation with the property $\left\|f-B_{k, N} f\right\|=O(\varepsilon), \varepsilon \rightarrow 0, k=1,2, \Rightarrow\left\|f-B_{N} f\right\|=O\left(\varepsilon^{2}\right) \|$. Operators of economic approximation have the form $\bar{B}_{n} f(x)=\left(\bar{B}_{1, N}+\bar{B}_{2, N}-B_{1, n} B_{2, N}\right) f(x)$, where the operators $\bar{B}_{k, N} f(x)$ can be obtained from the operators $B_{k, N} f(x)$ by the substitution $\Phi_{i_{k}, s_{k}}\left(x_{3-k}\right) \approx \varphi_{i_{k}, s_{k}}\left(x_{3-k}\right)$. Here $\Phi_{i_{k}, s_{k}}\left(x_{3-k}\right)$ are the operators of one-dimensional Fourier, Fejer, Bernstein, Haar, etc. approximation with the properties $\| \varphi_{i_{k}, s_{k}}\left(x_{3-k}\right)-$ $\Phi_{i_{k}, s_{k}}\left(x_{3-k}\right) \|=o\left(\varepsilon^{2}\right)$.

THEOREM 10. The approximation operators $\bar{B}_{N} f(x)$ use fewer values of Fourier coefficients, values of the function or Fourier-Haar coefficients than classical 2D operators $B_{1} B_{2} f(x)$ do (if they approximate $f(x)$ with the same accuracy $\left.O\left(\varepsilon^{2}\right)\right)$.

This result can be generalized to the case $n \geq 3$ for other operators $\bar{B}_{N}$.

\section{CONCLUSIONS}

The operators of interflation, interlineation, and blending approximation of functions of many variables considered in our review have the following properties.

1. The operators are constructed using traces of functions and (or) traces of some operators of the approximated function on a given system of lines/surfaces.

2. The interlineation and interflation operators have the same traces as the approximated function, which is a beneficial factor; though having very high accuracy, the operators of blending approximation do not generally coincide with the approximated function on the system of lines and (or) surfaces.

3. Constructed based on the operators of interlineation, interpolation, and blending approximation, the operators of interpolation and approximation using only the values of the approximated function are more accurate compared with the classical ones (when the same number of values of the approximated function is used).

4. The practice poses a wide range of problems with nonconventional experimental data. Their processing and efficient analysis are naturally involve the theory of interlineation and interflation operators.

5. Interlineation and interflation operators that employ traces of the approximated function and its normal derivatives up to an order $N \geq 1$ and preserve the differentiability class to which the approximated function belongs can form a basis for constructing better structures of approximate solutions to boundary-value problems, which require the approximating function to have continuous derivatives of a higher order.

The aforesaid means that the theory of approximation will be closely related to the interflation, interlineation, and blending approximation of functions of many variables. The domain of its applications will be extended. 


\section{REFERENCES}

1. J. H. Ahlberg, E. N. Nilson, and J. L. Walsh, The Theory of Splines and Their Applications, Acad. Press, New York (1967).

2. O. M. Anikeyenko, O. M. Lytvyn, V. L. Rvachov, and M. O. Safonov, "A formula for expansion in the neighborhood of an angle," Dop. URSR, Ser. A, No. 2, 99-101 (1972).

3. K. E. Babenko and O. M. Lytvyn, "The mathematical modeling in the computer tomography with using direct Fourier method and spline-interlineation of the functions," in: Proc. Int. Conf. ACIT-Signal and Image Processing (ACIT-SIP 2005), Novosibirsk, 20-24 June, 2005.

4. Yu. P. Vashchuk and O. M. Lytvyn, "Recovery of the structure of a 3D object based on tomograms in three systems of mutually perpendicular planes and blending approximation with Bernstein polynomials," in: Proc. Sci. Conf. UkrObraz'204 [in Ukrainian], Kyiv (2004), pp. 210-213.

5. L. I. Gulik and O. M. Lytvyn, "An approach to solving the Dirichlet boundary-value problem for the Poisson equation using interlineation,” Dop. NANU, No. 1, 13-18 (2004).

6. L. I. Gulik and O. M. Lytvyn, "A new approach to solving the Dirichlet boundary-value problem for the Poisson equation using interlineation of functions," Komp. Matem., No. 1 (2004).

7. L. I. Gulik and O. M. Lytvyn, "Interlineation of functions of three variables on a system of orthogonal straight lines," Dop. NANU, No. 11, 16-22 (2004).

8. L. I. Gulik, "Exact satisfaction of the boundary Dirichlet conditions on the boundary of a three-dimensional domain of complex form using interflation," in: Abstracts of Interstate Sci.-Method. Conf., Dneprodzerzhinsk, 26-28 May 2004 [in Russian] (2004), pp. 14-15.

9. L. I. Gulik and O. M. Lytvyn, "Exact satisfaction of boundary conditions for a three-dimensional domain of complex form using interflation," in: Proc. Intern. Conf. on Optimization of Computations (POO-XXXII) [in Ukrainian], Inst. Cybern., Kyiv (2005).

10. L. I. Gulik and O. N. Litvin, "Interflation of functions of three variables on pyramids with one curvilinear face," Cybern. Syst. Analysis, 41, No. 6, 818-831 (2005).

11. E. I. Drobot, O. M. Lytvyn, and I. V. Sergienko, "Numerical implementation of the LIDE method for the nonstationary heat-conduction equation with three spatial variables," Dop. NANU, No. 2, 67-73 (2000).

12. O. F. Kalaida, "On two-sided approximations of functions by polynomials," Vych. Prikl. Matem., Issue 67, 31-37 (1989).

13. V. V. Kamyshan, O. M. Lytvyn, and O. R. Maksymovych, "On numerical implementation of the interlineational method of finite elements for the Poisson equation in domains composed of rectangulars," Dop. NAN Ukr., Ser. A, No. 11, 34-38 (1995).

14. N. P. Korneichuk, Splines in the Theory of Approximation [in Russian], Nauka, Moscow (1984).

15. S. I. Kulik and O. M. Lytvyn, "Generalized Haar operators based on a two-dimensional blended approximation by Haar wavelets,” in: Proc. Sci. Conf. UkrObraz’2004 [in Ukrainian], Kyiv (2004), pp. 225-228.

16. S. I. Kulik and O. M. Lytvyn, "On numerical implementation of Haar wavelet transforms of one and two variables, constructed using experimental data," in: Proc. Intern. Conf. on Optimization of Calculations (POO-XXXII) [in Ukrainian], Kyiv (2005).

17. L. N. Kutsenko and O. N. Litvin, "Generalized interpolation on triangulated domains and its application in FEM," Dep. VINITI, January 13 1976, Pt. 1, No. 1379; February 20 1977, Pt. 2, No. 2303.

18. O. M. Lytvyn, Interlineation of Functions and Some Applications [in Ukrainian], Kyiv (2002).

19. O. N. Litvin, "Polynomial Taylor's interlineation of functions of two variables on several straight lines," Izv. Vuzov., Mat., No. 12, 19-27 (1989).

20. O. N. Litvin, "Polynomial interflation of functions of three variables on $M(M \geq 3)$ intersected planes without preserving the class $C^{r}\left(R^{3}\right), "$ Dokl. AN USSR, No. 11, 35-41 (1991).

21. O. M. Lytvyn, "Interlineation and interflation functions of many variables (blending function interpolation) and economical algorithms in the approximation theory," in: Proc. Int. Conf. Comput. Methods, ICCM-2004, Singapour, 15-17 Dec. (2004). 
22. O. M. Lytvyn, "Interlineation and interflation functions of many variables (the blending function interpolation) and multidimensional signal processing," Int. Conf. ACIT-Signal and Image Processing (ACIT-SIP 2005), Novosibirsk, 20-24 June (2005).

23. O. M. Lytvyn, Computational Methods. Additional Sections: A Textbook [in Ukrainian], Naukova Dumka, Kyiv (2005).

24. O. M. Lytvyn and O. P. Nechuiviter, "Computing Fourier coefficients using formulas of spline interlineation with splines of the second order,” in: Proc. Intern. Sci. Conf. UkrObraz'2004 [in Ukrainian], Kyiv (2004), pp. $214-217$.

25. O. M. Lytvyn and V. V. Mikhalkin, "Computing Fourier coefficients using Radon data based on the Filon formula," in: Proc. Intern. Sci. Conf. UkrObraz’2004 [in Ukrainian], Kyiv (2004), pp. 205-209.

26. O. M. Lytvyn and V. V. Mikhalkin, "Approximate solution of a problem of X-ray and computer-aided tomography by using piecewise-constant splines," in: Proc. Intern. Conf on Optimization of Computations (POO-XXXII) [in Ukrainian], Kyiv (2005).

27. O. N. Litvin and V. A. Pasechnik, "Optimizing a mathematical model of a 3D body surface," Cybern. Syst. Analysis, 42, No. 1, 90-97 (2006).

28. O. M. Lytvyn and Yu. I. Pershina, "Recovering 3-D objects from their traces on a system of intersecting planes using interflation of functions,” in: Proc. Intern. Sci. Conf. UkrObraz'2004 [in Ukrainian], Kyiv (2004), pp. 221-224.

29. O. Lytvyn and Yu. Pershina, "Reconstruction of three-dimensional objects under their tomograms on system of three groups of the crossed planes with use interflation of functions," in: Proc. Int. Conf. ACIT-Signal and Image Processing (ACIT-SIP 2005), Novosibirsk, 20-24 June (2005).

30. O. M. Lytvyn and V. M. Udovichenko, "Operators of two-dimensional finite discrete-continuous Fourier transform that are based on piecewise-constant splines and exact on trigonometric polynomials of prescribed order," in: Vestn. NTU "KhPI", Special Issue: Power and Thermal Processes and Equipment, 9'2003, Vol. 2 [in Ukrainian], Kharkov (2003), pp. 108-117.

31. O. M. Lytvyn and V. M. Udovichenko, "Operators of two-dimensional finite discrete-continuous Fourier transform that are based on first-order splines and exact on trigonometric polynomials of prescribed order," in: Vestn. NTU “KhPI”, Special Issue: Radiophysics and Ionosphere, 7'2003, Vol. 4 [in Ukrainian], Kharkov (2003), pp. 49-58.

32. O. M. Lytvyn and V. M. Udovichenko, "Operators of two-dimensional finite discrete-continuous Hartley transform that are based on piecewise-constant splines and exact on trigonometric polynomials of prescribed order," in: Vestn. NTU "KhPI", Special Issue: Automation and Instrumentation, 7’2003, Vol. 3 [in Ukrainian], Kharkov (2003), pp. 105-114.

33. O. M. Lytvyn and V. M. Udovichenko, "Three-dimensional finite Fourier and Hartley transforms using interflation of functions," in: Vestn. NTU “KhPI”, Special Issue: Automation and Instrumentation, 16'2005 [in Ukrainian], Kharkov (2005), pp. 117-157.

34. O. M. Lytvyn and V. M. Udovichenko, "A generalization of the concept of trend for nonperiodic functions of two variables,” in: Proc. Sci. Conf. UkrObraz’2004 [in Ukrainian], Kyiv (2004), pp. 217-220.

35. O. M. Lytvyn and V. M. Udovichenko, "Operators of finite three-dimensional discrete-continuous Hartley transform that are based on the Filon method and three-linear splines and exact on trigonometric polynomials of a prescribed order," in: Radioelectronic and Computer Systems [in Ukrainian], KhAI, Kharkov, No. 2 (6), April-June (2004), pp. $123-127$.

36. O. M. Lytvyn and V. M. Udovichenko, "Operators of finite three-dimensional discrete-continuous Hartley transform that are based on the Filon method and piecewise-constant splines and exact on trigonometric polynomials of a prescribed order,” in: Vestn. NTU “KhPI,” Special Issue: Dynamics and Strength of Machines, 19’2004 [in Ukrainian], Kharkov (2004), pp. 133-140.

37. O. M. Lytvyn and V. M. Udovichenko, "Two-dimensional finite Fourier and Hartley transforms using interlineation of functions," in: Vestn. NTU “KhPI," Special Issue: Automation and Instrumentation, 7’2005 [in Ukrainian], Kharkov (2005), pp. 100-111.

38. O. M. Lytvyn and V. M. Udovichenko, "Operators of finite three-dimensional Fourier transform," Radioelektronika Informatika, No. 4 (29), 130-133 (2004).

39. O. M. Lytvyn and V. M. Udovichenko, "Operators of finite three-dimensional discrete-continuous Fourier transform that are based on the Filon method and three-linear splines and are exact on trigonometric polynomials of prescribed order," in: Inform.-Keruyuchi Systemy na Zalizn. Transporti, 1, 2 (51, 52), 19-23 (2005). 
40. O. M. Lytvyn and V. M. Udovichenko, "Three-dimensional Fourier and Hartley transforms using interflation of functions in: Vestn. NTU "KhPI," Special Issue: Automation and Instrumentation, 38, No. 5 (2005).

41. O. N. Litvin and V. V. Fed'ko, "Generalized piecewise-Hermitian interpolation," Ukr. Mat. Zh., 28, No. 6, 812-819 (1976).

42. O. N. Litvin and V. V. Fed'ko, "On a solution of the first boundary-value problem for the biharmonic equation using the Hermite generalized interpolation,” Dep. VINITI, Sept. 21, 1976, No. 4329.

43. A. M. Pidgornyi and O. M. Lytvyn, "A method of solving boundary-value problems for partial derivative equations by reducing them to systems of ordinary integrodifferential equations," Dop. AN URSR, Ser. A, No. 6, 522-525 (1974).

44. V. L. Rvachev, Geometrical Application of Logic Algebra [in Russian], Tekhnika, Kyiv (1967).

45. I. V. Sergienko and O. M. Lytvyn, "Numerical implementation the LIDE method for a heat-conduction equation," Dop. AN URSR, Ser. A, No. 10, 69-73 (1990).

46. J. C. Cavendish, W. J. Gordon, and C. A. Hall, "Ritz-Galerkin approximation in blending function spaces," Numer. Mathem., 26, No. 2, 155-178 (1976).

47. S. A. Coons, "Surface for computer-aided design of space forms," in: Project MAC report MAC-TR-41, Cambridge, June (1967), pp. 3-30.

48. W. Haussmann, K. Getter, and B. Steinhaus, "Degree of best approximation by trigonometric blending functions," Math. Ztschr., 189, No. 1, 143-150 (1985).

49. W. Haussmann and K. Zeller, "Blending interpolation and best $L_{1}$-approximation," Arch. Math. (Basel), 40, No. 6, 545-552 (1983).

50. D. I. Mangeron, "Sopra un problema al contorno per un'equatione differentiable alle derivate parziali di quartordine conle caratteristice reali dopie," Rend. Accad. Sci. Fis. Mat. Napoli., No. 2, $28-40$ (1932). 